\title{
Erratum to: Ethical standards for mental health and psychosocial support research in emergencies: review of literature and current debates
}

\author{
Anna Chiumento ${ }^{1 *}$, Atif Rahman ${ }^{1}$, Lucy Frith' ${ }^{1}$ Leslie Snider ${ }^{2}$ and Wietse A. Tol ${ }^{3}$
}

\section{Erratum}

In the original publication [1] of this article on 8 February 2017 there are 3 errors related to the citations and their corresponding references. In this Erratum the correct and incorrect citations are displayed in bold. The page numbers for these citations were correct in the original publication. The citations in the original publication of this article have been updated.

The first incorrect reference \& citation was in the below section:

1. Consent as "informed" is defined universally as: "an understanding of study purpose, who are the targeted beneficiaries, and the implications of involvement...information is communicated in a form appropriate to the culture, age, and educational level of that individual" [14 - p.s224].

a. The above citation originally referred to: [14] World Health Organisation. WHO Ethical and Safety Recommendations for Researching, Documenting and Monitoring Sexual Violence in Emergencies. Geneva: WHO; 2007. p. 1-33.

The correct citation belonging to this sentence is:

- [21] Allden K, et al. Mental health and psychosocial support in crisis and conflict: report of the Mental Health Working Group. Prehospital And Disaster Medicine. 2009;24(Suppl 2):s217-27.

The second incorrect reference \& citation was in the below section:

* Correspondence: Anna.Chiumento@liverpool.ac.uk

${ }^{1}$ University of Liverpool, Institute of Psychology, Health and Society, 2nd Floor, Block B, Waterhouse Building, 1-5 Brownlow Street, L69 3GL, Liverpool, UK

Full list of author information is available at the end of the article
2. Despite the challenges, the researcher's duty to safeguard privacy and confidentiality both during and after research is highlighted $[15,18,27]$ : "anyone asking someone to disclose information bears a responsibility to safeguard that information" [18 - p.18].

b. The above citation originally referred to: [18] O'Mathuna DP. Conducting research in the aftermath of disasters: ethical considerations. J Evid Based Med. 2010;3(2):65-75.

The correct citation belonging to this sentence is:

- [14] World Health Organisation. WHO Ethical and Safety Recommendations for Researching, Documenting and Monitoring Sexual Violence in Emergencies. Geneva: WHO; 2007. p. 1-33.

The third incorrect reference \& citation was in the below section:

3. To achieve informed consent there are calls for moving away from procedural, juridical and ritualised consent, avoiding "a crude version of the biomedical model of consent: The dialogue should not be seen as merely ... making the informant understand and accept a pre-defined research package" [59 - p.1746].

c. The above citation originally referred to: [59] Yamout R, Jabbour S. Complexities of research during war: lessons from a survey conducted during the summer 2006 war in Lebanon. Public Health Ethics. 2010;3(3):293-300.

The correct citation belonging to this sentence is: 
- [60] Hoeyer K, Dahlager L, Lynoe N. Conflicting notions of research ethics. The mutually challenging traditions of social scientists and medical researchers. Soc Sci Med.

2005;61(8):1741-9.

Author details

${ }^{1}$ University of Liverpool, Institute of Psychology, Health and Society, 2nd

Floor, Block B, Waterhouse Building, 1-5 Brownlow Street, L69 3GL, Liverpool,

UK. ${ }^{2}$ Independent Consultant, Peace in Practice, Amsterdam, The

Netherlands. ${ }^{3}$ Johns Hopkins University, School of Public Health and The

Peter C. Alderman Foundation, Baltimore, USA.

Published online: 29 June 2017

\section{Reference}

1. Chiumento et al. (2017) Ethical standards for mental health and

psychosocial support research in emergencies: review of literature and current debates. 13:8. doi: 10.1186/s12992-017-0231-y 